Article

\title{
New Route Synthesis of Thiadiazoles, Bisthiadiazoles, Thiadiazolotriazines, and Pyrazolothiadiazoles Based on Hydrazonoyl Halides and Dihydrazinylthiadiazole
}

\author{
Abdelwahed R. Sayed ${ }^{1,2, *}$ and Shar Saad Al-Shihry ${ }^{1}$ \\ 1 Department of Chemistry, Faculty of Science, King Faisal University, Hofuf 31982, Saudi Arabia; \\ sshihry@kfu.edu.sa \\ 2 Department of Chemistry, Faculty of Science, Beni-Suef University, Beni-Suef 62514, Egypt \\ * Correspondence: arsayed@kfu.edu.sa
}

Academic Editor: Panayiotis A. Koutentis

Received: 1 January 2017; Accepted: 15 February 2017; Published: 21 February 2017

\begin{abstract}
Synthesis and characterization of new thiadiazoles, bisthiadiazoles from the reaction of mono- and di-hydrazonoyl halides with various hydrazinecarbodithioate derivatives were studied. Treatment of hydrazonoyl halides with 2,5-dihydrazinyl-1,3,4-thiadiazole afforded new bistriazines containing thiadiazole; we also examined the reaction of 2,5-dihydrazinyl-1,3,4-thiadiazole with active methylene compounds to afford new pyrazoles containing thiadiazole compounds. The new synthesized compounds were identified by elemental analysis and various spectral data (Fourier transform infrared spectroscopy, mass spectrometry, ${ }^{1} \mathrm{H}$ and ${ }^{13} \mathrm{C}$ nuclear magnetic resonance).
\end{abstract}

Keywords: hydrazonoyl; dihydrazinylthiadiazole; hydrazinecarbodithioate; thiadiazoles

\section{Introduction}

Hydrazonoyl halides are useful for the synthesis of assorted and various heterocyclic derivatives [1-4]. Treatment of hydrazonoyl halides with dithioate derivatives in dioxane and in the presence of base gave the thiadiazole derivatives [5]. Thiadiazoles are heterocyclic organic compounds with a comprehensive range of biological activities, such as anticancer [6], antivirus [7], antimicrobial [8], and anti-inflammatory [9]. Heterocycles are used in analytical chemistry [10] and have pharmaceutical properties [11]. Thiadiazoles are described and quantum chemistry is used to elucidate the chemical reactions in [12]. Studying the aromaticity of thiadiazoles via various quantitative methods is reported in $[13,14]$.

2,5-Dimercapto-1,3,4-thiadiazole derivatives and lubricants containing them are reported in [15]. Thiadiazoles are synthesized to study biological activity in [16,17]. Thiadiazoles carrying electron-donating methyl or hydroxy on one or both of the rings have been studied in complexation with different metals [18]. Thiadiazoles are prepared for use in medicinal chemistry [19-21]. Diaminothiadiazoles reacted with diacidchlorides gave polyamides compounds in [22]. Treatment oxadiazolylphenylthiourea with hydrazonoyl halides gave oxadiazolylimino thiadiazoles [23].

Ferrocene is used as a multi-nuclear substance possessing the properties of both organometlallic and coordination complexes.

Herein, we synthesized thiadiazoles, bisthiadiazoles, thiadiazolotriazines and pyrazolothiadiazoles based on hydrazonoyl halides and 2,5-dihydrazinyl-1,3,4-thiadiazole. The new final products will be identified by different techniques, such as elemental analysis, Fourier transform infrared spectroscopy (FT-IR), nuclear magnetic resonance (NMR), mass spectrometry (MS), and alternate synthesis whenever possible. 


\section{Results and Discussion}

This work is a continuation of our active research in the area of hydrazonoyl halides and their reactions with different moieties, reported in $[2,3]$. These principles were extended in the present paper. Thus, hydrazonoyl halide 1 [24] was reacted with methyl hydrazinecarbodithioate 2 [25] in ethanol and in the presence of triethylamine under heating until complete elimination of methanethiol. The reaction mixture gave a single isolated product in each case 5a-e monitored by thin layer chromatography (TLC). The formation of the final products can be explained by stepwise mechanism involving nucleophilic substitution reaction to give acyclic thiohydrazonate ester 3, which undergoes intramolecular cyclization to yield the spirothiadiazole intermediate 4, which was followed by elimination of methanethiol in order to give the final products 5a-e or via 1,3-dipolar cycloaddition of nitrilimine (generated in situ from hydrazonoyl halides in the presence of triethylamine) to $C=S$ of $\mathbf{2}$, which was followed by elimination of methanethiol to give the final products $5 \mathbf{a}-\mathbf{e}$. The final products were elucidated on the basis of spectral data and elemental analysis as depicted in Scheme 1. The infrared (IR) spectrum of 5a-e showed absorption bands for $\mathrm{NH}_{2}$ group around $3333-3220 \mathrm{~cm}^{-1}$. In addition, the ${ }^{1} \mathrm{H}-\mathrm{NMR}$ spectrum for 5 showed signals attributed to the $\mathrm{NH}_{2}$ protons $5.72-5.70 \mathrm{ppm}$, as depicted in Scheme 1.

Treatment of hydrazonoyl halides 1 with methyl 2-(1-ferrocenylethylidene)hydrazine-carbodithioate 8 [26] in boiling ethanol/dimethylformamide in the presence of triethylamine under reflux afforded 7. The assigned structure 2-(1-ferrocenylethylidenehydrazono)-3-(4-bromophenyl)-5methoxyphenyl)-2,3-dihydro-1,3,4-thiadiazole 7 was further confirmed by alternate synthesis via the reaction of thiadiazoles 5 with the acetylferrocene 6 in ethanol, giving thiadiazole 7, and was an identical product in all respects ( $\mathrm{mp}$, mixed $\mathrm{mp}$, and spectra) (Scheme 1) with that obtained above from the reaction of $1 \mathrm{a}$ with 8 .

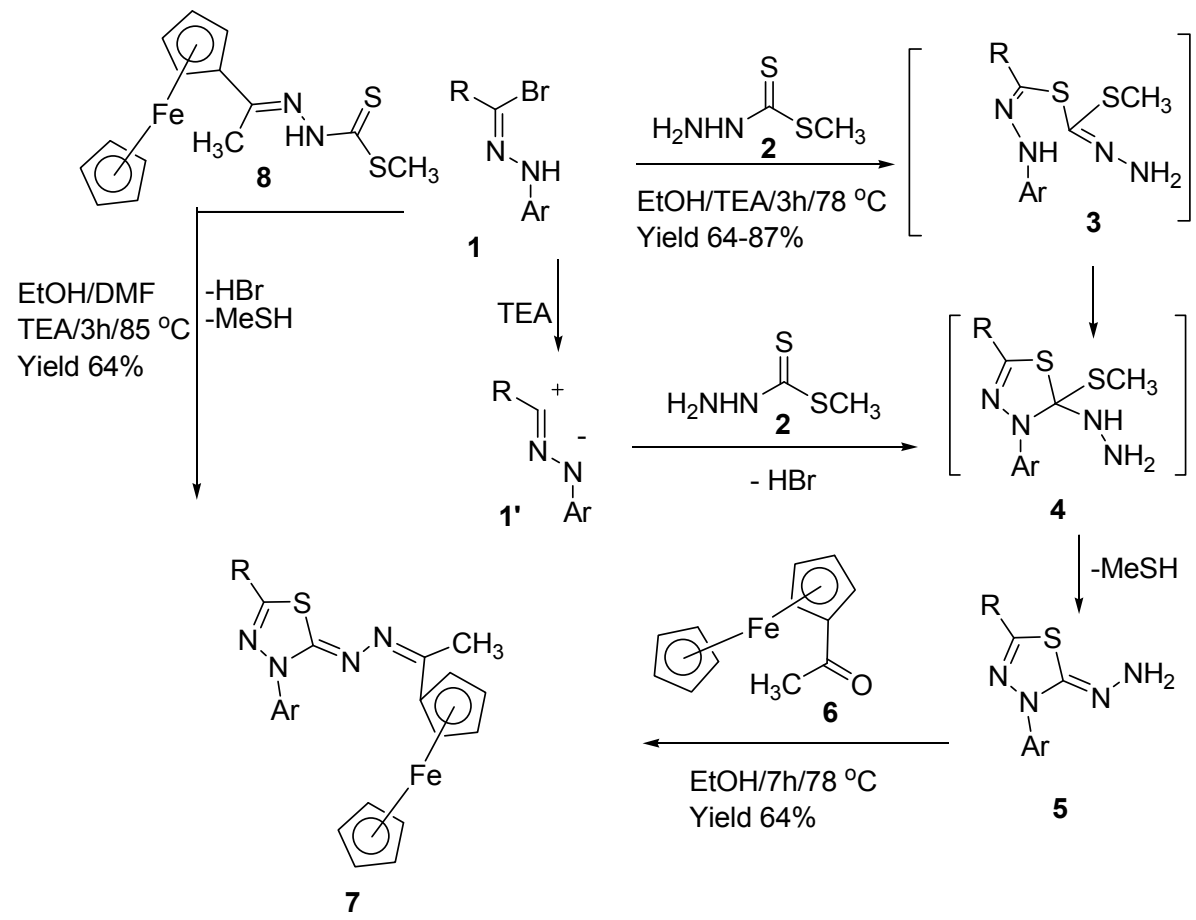

R: a, 4- $\mathrm{CH}_{3} \mathrm{OC}_{6} \mathrm{H}_{4} ; \mathrm{b}, 4-\mathrm{CH}_{3} \mathrm{C}_{6} \mathrm{H}_{4} ; \mathrm{c}, \mathrm{C}_{6} \mathrm{H}_{5} ; \mathrm{d}, 4-\mathrm{ClC}_{6} \mathrm{H}_{4} ; \mathrm{e}, 4-\mathrm{BrC}_{6} \mathrm{H}_{4}$

Ar: $4-\mathrm{BrC}_{6} \mathrm{H}_{4}$

Scheme 1. Synthesis of thiadiazole derivatives. TEA: triethylamine. 
Analogously, novel compounds $\mathbf{1 1}$ and $\mathbf{1 2}$ were prepared via nucleophilic substitution reaction of 1,4-diphenylterephthalohydrazonoyl dichloride 9 [27] with methyl 2-(1-ferrocenylethylidene) hydrazinecarbodithioate 8 or methyl 2-(1-phenylethylidene)hydrazinecarbodithioate 10 [28] in $\mathrm{EtOH} / \mathrm{DMF}$ and in the presence of triethylamine acting as base to give the final products $\mathbf{1 1}$ and $\mathbf{1 2}$ in good yields, as depicted in Scheme 2. The final products $\mathbf{1 1}$ and $\mathbf{1 2}$ gave a satisfactory elemental analysis and spectroscopic data (IR, NMR, and MS) consistent with their assigned structures (Scheme 2). The IR spectra of products $\mathbf{1 1}$ and $\mathbf{1 2}$ indicate the absence of $\mathrm{NH}$ at $3300 \mathrm{~cm}^{-1}(\mathrm{NH})$.

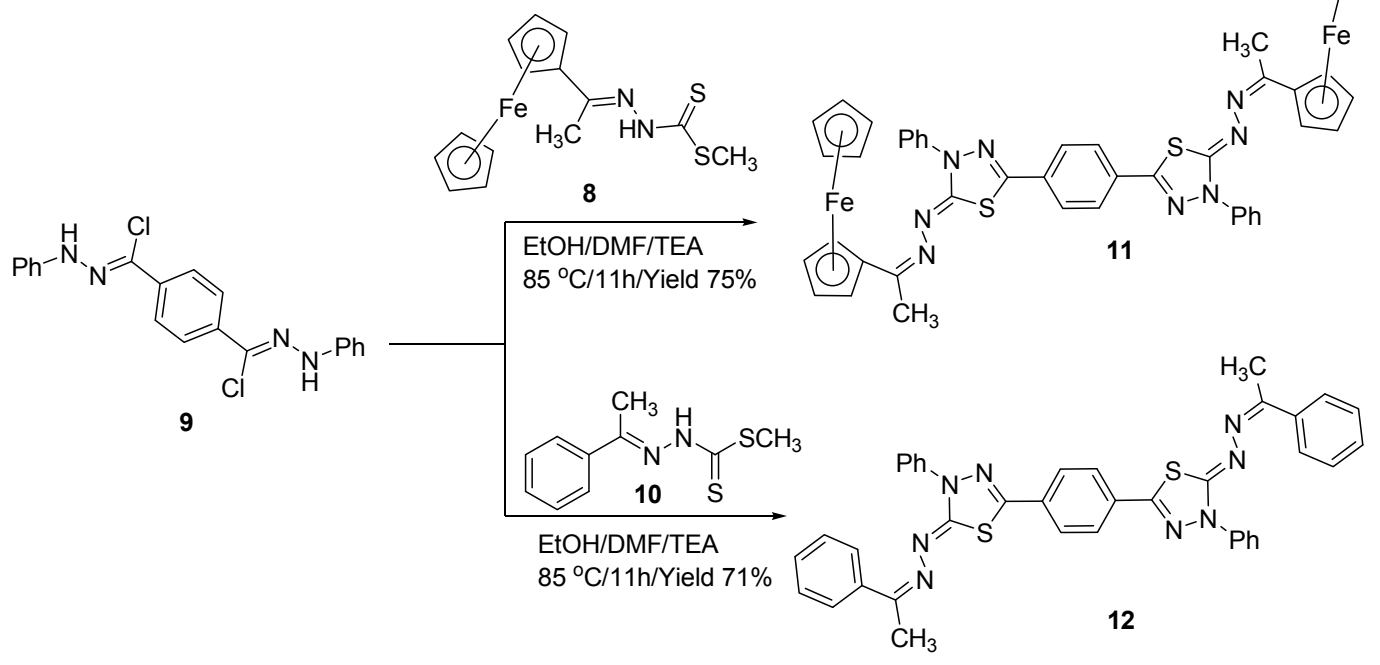

Scheme 2. Synthesis of bisthiadiazole derivatives.

Pleasingly, reaction of 2,5-dihydrazinyl-1,3,4-thiadiazole 13 [29] with hydrazonoyl bromide 14 [30] in dioxane in the presence of trimethylamine as a base under reflux conditions proceeded smoothly to afford 17; it is suggested that the reaction starts with the formation of hydrazide $\mathbf{1 5}$ followed by cyclization to give the product $\mathbf{1 7}$ via elimination of water molecule as depicted in Scheme 3 . The compounds were characterized by elemental analysis and spectral data (IR, MS, ${ }^{1} \mathrm{H}-\mathrm{NMR}$ spectra).

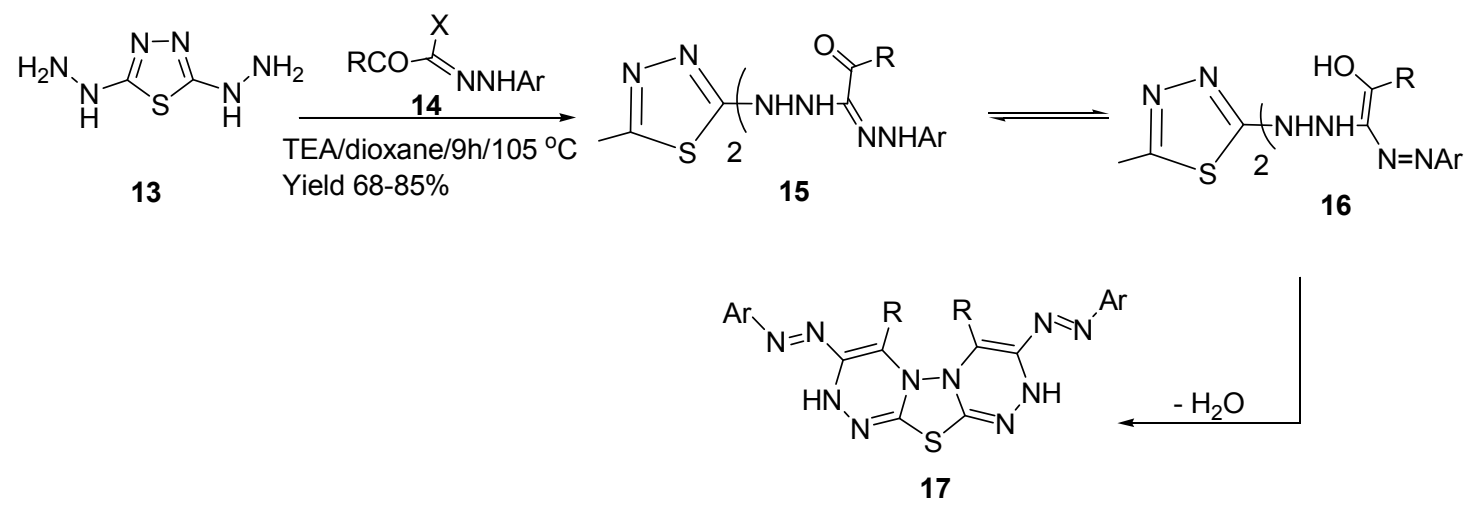

$\mathrm{R}=\mathrm{Me}, \mathrm{Ph}$

Ar : a, $4-\mathrm{CH}_{3} \mathrm{C}_{6} \mathrm{H}_{4} ; \mathrm{b}, \mathrm{C}_{6} \mathrm{H}_{5} ; \mathrm{c}, 4-\mathrm{ClC}_{6} \mathrm{H}_{4}$

Scheme 3. Synthesis of thiadiazolotriazines.

Finally, our study was extended to the reaction of 2,5-dihydrazinyl-1,3,4-thiadiazole 13 with acetylacetone $\mathbf{1 8}$ or ethyl acetoacetate 19 in glacial acetic acid to synthesize compounds 22 and 23, 
respectively (Scheme 4). The structures of final products 22 and 23 were confirmed on the basis of spectroscopic data and elemental analyses (see Experimental Section).

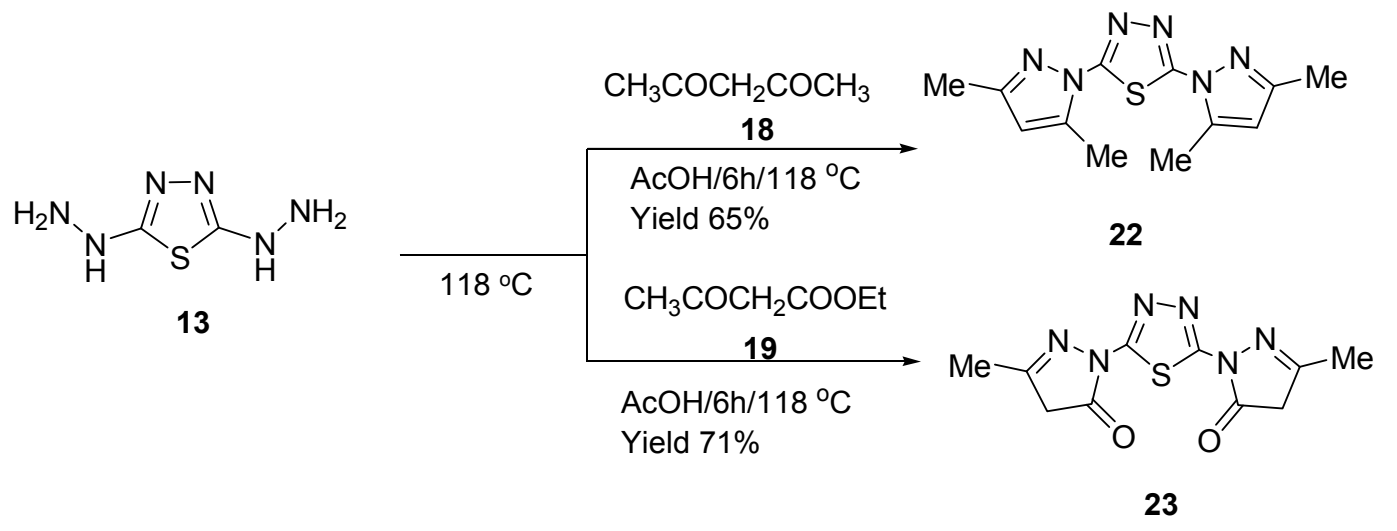

Scheme 4. Synthesis of pyrazolothiadiazoles.

\section{Experimental Section}

All melting points were determined on an electrothermal apparatus and are uncorrected. IR spectra were recorded (KBr discs) on a Shimadzu FT-IR 8201 PC spectrophotometer (Shimadzu, Tokyo, Japan). ${ }^{1} \mathrm{H}-\mathrm{NMR}$ spectra were recorded in $\mathrm{CDCl}_{3}$ and $\left(\mathrm{CD}_{3}\right)_{2} \mathrm{SO}$ solutions on a Varian Gemini $300 \mathrm{MHz}$ spectrometer (Agilent, Palo Alto, CA, USA), and chemical shifts are expressed in $\delta$ units using tetramethylsilane (TMS) as an internal reference. Mass spectra were recorded on a Shimadzu GC-MS QP 1000 EX instrument. Elemental analyses were carried out at the Microanalytical Canter of Cairo University.

\subsection{Synthesis of Thiadiazoles (5a-e)}

To a solution of hydrazonoyl bromides $1 \mathrm{a}-\mathbf{e}(5 \mathrm{mmol})$ and methyl hydrazinecarbodithioate 2 $(0.61 \mathrm{~g}, 5 \mathrm{mmol})$ in ethanol $(40 \mathrm{~mL})$ was added triethylamine (TEA) $(5 \mathrm{mmol}, 0.7 \mathrm{~mL})$, and the mixture was refluxed for $3 \mathrm{~h}$ monitored by TLC. The resulting solids were collected and recrystallized from an appropriate solvent to give final products $5 \mathbf{a}-\mathbf{e}$.

3-(4-Bromophenyl)-2-hydrazono-5-(4-methoxyphenyl)-2,3-dihydro-1,3,4-thiadiazole (5a). Pale green crystalline solid from EtOH/DMF, Yield (69\%); m.p. $198{ }^{\circ} \mathrm{C}$; IR $\left(\mathrm{cm}^{-1}\right)(\mathrm{KBr}): 3315,3180\left(\mathrm{NH}_{2}\right), 1604(\mathrm{C}=\mathrm{N})$; ${ }^{1} \mathrm{H}-\mathrm{NMR}\left(300 \mathrm{MHz}, \mathrm{DMSO}-d_{6}\right): \delta 3.73\left(\mathrm{~s}, 3 \mathrm{H}, \mathrm{OCH}_{3}\right), 5.72\left(\mathrm{~s}, 2 \mathrm{H}, \mathrm{NH}_{2}\right), 6.49-7.84(\mathrm{~m}, 8 \mathrm{H}, \mathrm{ArH}) \mathrm{ppm}$. Anal. Calcd. for $\mathrm{C}_{15} \mathrm{H}_{13} \mathrm{BrN}_{4} \mathrm{OS}$ : C, 47.76; H, 3.47; N, 14.85. Found: $\mathrm{C}, 47.72 ; \mathrm{H}, 3.49 ; \mathrm{N}, 14.81 \%$.

3-(4-Bromophenyl)-2-hydrazono-5-(p-tolyl)-2,3-dihydro-1,3,4-thiadiazole (5b). Pale green crystalline solid from EtOH/DMF, Yield (64\%); m.p. $177^{\circ} \mathrm{C}$; IR $\left(\mathrm{cm}^{-1}\right)(\mathrm{KBr}): 3300,3205\left(\mathrm{NH}_{2}\right), 1601(\mathrm{C}=\mathrm{N}) ;{ }^{1} \mathrm{H}-\mathrm{NMR}$ $\left(300 \mathrm{MHz}, \mathrm{DMSO}-d_{6}\right): \delta 2.34\left(\mathrm{~s}, 3 \mathrm{H}, \mathrm{CH}_{3}\right), 5.72\left(\mathrm{~s}, 2 \mathrm{H}, \mathrm{NH}_{2}\right), 6.71-7.81(\mathrm{~m}, 8 \mathrm{H}, \mathrm{ArH}) ;{ }^{13} \mathrm{C}-\mathrm{NMR}$ (300 MHz, DMSO- $\left.d_{6}\right): \delta 21.43,126.47,127.77,129.48,130.33,132.53,136.312,140.53,141.63,150.75$, and 154.91 ppm. Anal. Calcd. for $\mathrm{C}_{15} \mathrm{H}_{13} \mathrm{BrN}_{4} \mathrm{~S}: \mathrm{C}, 49.87 ; \mathrm{H}, 3.63 ; \mathrm{N}$, 15.51. Found: $\mathrm{C}, 49.89 ; \mathrm{H}, 3.61$; $\mathrm{N}, 15.53 \%$.

3-(4-Bromophenyl)-2-hydrazono-5-phenyl-2,3-dihydro-1,3,4-thiadiazole (5c). Pale yellow crystalline solid from EtOH/DMF, Yield (73\%); m.p. $179^{\circ} \mathrm{C}$; IR $\left(\mathrm{cm}^{-1}\right)(\mathrm{KBr}): 3333,3210\left(\mathrm{NH}_{2}\right), 1600(\mathrm{C}=\mathrm{N}) ;{ }^{1} \mathrm{H}-\mathrm{NMR}$ (300 MHz, DMSO- $\left.d_{6}\right): \delta 5.71\left(\mathrm{~s}, 2 \mathrm{H}, \mathrm{NH}_{2}\right), 6.78-7.91(\mathrm{~m}, 9 \mathrm{H}, \mathrm{ArH}) \mathrm{ppm}$. Anal. Calcd. for $\mathrm{C}_{14} \mathrm{H}_{11} \mathrm{BrN}_{4} \mathrm{~S}$ : C, 48.43; H, 3.19; N, 16.14. Found: C, 48.45; H, 3.21; N, 16.11\%.

3-(4-Bromophenyl)-2-hydrazono-5-(4-chlorophenyl)-2,3-dihydro-1,3,4-thiadiazole (5d). Pale yellow crystalline solid from EtOH/DMF, Yield (87\%); m.p. $183{ }^{\circ} \mathrm{C}$; IR $\left(\mathrm{cm}^{-1}\right)(\mathrm{KBr}): 3300,3222\left(\mathrm{NH}_{2}\right), 1610(\mathrm{C}=\mathrm{N})$; ${ }^{1} \mathrm{H}-\mathrm{NMR}\left(300 \mathrm{MHz}, \mathrm{DMSO}-d_{6}\right): 5.70\left(\mathrm{~s}, 2 \mathrm{H}, \mathrm{NH}_{2}\right), 6.81-7.96(\mathrm{~m}, 8 \mathrm{H}, \mathrm{ArH}) \mathrm{ppm}$. Anal. Calcd. for $\mathrm{C}_{14} \mathrm{H}_{10} \mathrm{BrClN}_{4} \mathrm{~S}: \mathrm{C}, 44.06 ; \mathrm{H}, 2.64 ; \mathrm{N}, 14.68$. Found: $\mathrm{C}, 44.09 ; \mathrm{H}, 2.68 ; \mathrm{N}, 14.65 \%$. 
3-(4-Bromophenyl)-2-hydrazono-5-(4-bromophenyl)-2,3-dihydro-1,3,4-thiadiazole (5e). Pale green crystalline solid from EtOH/DMF, Yield (81\%); m.p. $145^{\circ} \mathrm{C}$; IR $\left(\mathrm{cm}^{-1}\right)(\mathrm{KBr}): 3305,3200\left(\mathrm{NH}_{2}\right), 1604(\mathrm{C}=\mathrm{N})$; ${ }^{1} \mathrm{H}-\mathrm{NMR}\left(300 \mathrm{MHz}\right.$, DMSO- $\left.d_{6}\right): \delta 5.70\left(\mathrm{~s}, 2 \mathrm{H}, \mathrm{NH}_{2}\right), 6.81-8.01(\mathrm{~m}, 8 \mathrm{H}, \mathrm{ArH}) \mathrm{ppm}$. Anal. Calcd for $\mathrm{C}_{14} \mathrm{H}_{10} \mathrm{Br}_{2} \mathrm{~N}_{4} \mathrm{~S}: \mathrm{C}, 39.46 ; \mathrm{H}, 2.37 ; \mathrm{N}, 13.15$. Found: $\mathrm{C}, 39.44 ; \mathrm{H}, 2.39 ; \mathrm{N}, 13.19 \%$.

3.2. Synthesis of 2-(1-Ferrocenylethylidenehydrazono-3-(4-bromophenyl)-5-methoxyphenyl)-2,3-dihydro1,3,4-thiadiazole (7)

Method A: An equimolar amount of the appropriate hydrazonoyl bromides 1a (5 mmol, $1.135 \mathrm{~g}$ ) and methyl 2-(1-ferrocenylethylidene)hydrazinecarbodithioate 8 (5 mmol, $1.645 \mathrm{~g}$ ) in $10 \mathrm{~mL} \mathrm{DMF}$, $30 \mathrm{~mL}$ ethanol, triethylamine $(5 \mathrm{mmol}, 0.7 \mathrm{~mL}$ ); the reaction mixtures were refluxed for $7 \mathrm{~h}$ at boiling point monitored by TLC. The solvent was evaporated, and the residue was triturated with methanol. The formed solid was filtered and recrystallized from appropriate to give compounds 7.

Method B: An equimolar amount of the appropriate 1,3,4-thiadiazoline derivatives 5 a $(5 \mathrm{mmol}$, $1.885 \mathrm{~g})$ and acetylferrocene $6(5 \mathrm{mmol}, 0.113 \mathrm{~g})$ in absolute ethanol $(40 \mathrm{~mL})$ were refluxed for $7 \mathrm{~h}$. The resulting solids were collected by cooling and crystallized from an appropriate solvent to give $\mathbf{7}$.

2-(1-Ferrocenylethylidenehydrazono)-3-(4-bromophenyl)-5-methoxyphenyl)-2,3-dihydro-1,3,4-thiadiazole (7). Brown crystalline solid from EtOH/DMF; Yield (64\%); m.p. $175^{\circ} \mathrm{C} ; \mathrm{IR}\left(\mathrm{cm}^{-1}\right)(\mathrm{KBr}): 3081,3055(\mathrm{C}-\mathrm{H}$ Ar), $1615(\mathrm{C}=\mathrm{N}) ;{ }^{1} \mathrm{H}-\mathrm{NMR}\left(300 \mathrm{MHz}, \mathrm{DMSO}-d_{6}\right): \delta 2.51\left(\mathrm{~s}, 3 \mathrm{H}, \mathrm{CH}_{3}\right), 3.72\left(\mathrm{~s}, 3 \mathrm{H}, \mathrm{OCH}_{3}\right), 4.18(\mathrm{~m}, 5 \mathrm{H}$, $\left.\mathrm{C}_{5} \mathrm{H}_{5}\right), 4.64\left(\mathrm{~m}, 4 \mathrm{H}, \mathrm{C}_{5} \mathrm{H}_{4}\right), 6.99-8.32\left(\mathrm{~m}, 8 \mathrm{H}, \mathrm{ArH}^{\prime} \mathrm{s}\right)$ ppm. Anal. Calcd. for $\mathrm{C}_{27} \mathrm{H}_{23} \mathrm{BrFeN}_{4} \mathrm{OS}: \mathrm{C}, 55.22$; $\mathrm{H}, 3.95 ; \mathrm{N}, 9.54$. Found: $\mathrm{C}, 55.19 ; \mathrm{H}, 3.91 ; \mathrm{N}, 9.56 \%$.

\subsection{Synthesis of Bisthiadiazoles (11) and (12)}

An equimolar amount of the appropriate 1,4-diphenylterephthalohydrazonoyl dichloride 9 (5 mmol, $1.91 \mathrm{~g}$ ) and methyl 2-(1-ferrocenylethylidene)hydrazinecarbodithioate 8 (10 mmol, $3.29 \mathrm{~g})$ or methyl 2-(1-phenylethylidene)hydrazinecarbodithioate 10 (10 mmol, $2.24 \mathrm{~g})$ in ethanol/DMF $(30 / 10 \mathrm{~mL})$ was added triethylamine $(10 \mathrm{mmol}, 1.4 \mathrm{~mL})$, dropwise were refluxed for $11 \mathrm{~h}$ at boiling point. The solvent was evaporated and the residue was triturated with methanol. The formed solid was filtered and recrystallized from appropriate solvent to give compounds $\mathbf{1 1}$ and $\mathbf{1 2}$.

1,4-Bis-(5-(1-ferrocenylethylidenehydrazono)-4-phenyl-4,5-dihydro-1,3,4-thiadiazol-2-yl)benzene (11). Brown crystalline solid from EtOH/DMF; Yield (75\%); $>300{ }^{\circ} \mathrm{C}$; IR $\left(\mathrm{cm}^{-1}\right)(\mathrm{KBr}): 3081,3055(\mathrm{C}-\mathrm{H} \mathrm{Ar}), 1609$ $(\mathrm{C}=\mathrm{N}) ;{ }^{1} \mathrm{H}-\mathrm{NMR}\left(300 \mathrm{MHz}, \mathrm{DMSO}-d_{6}\right): \delta 2.52\left(\mathrm{~s}, 6 \mathrm{H}, 2 \mathrm{CH}_{3}\right), 4.18\left(\mathrm{~m}, 10 \mathrm{H}, 2 \mathrm{C}_{5} \mathrm{H}_{5}\right), 4.64\left(\mathrm{~m}, 8 \mathrm{H}, 2 \mathrm{C}_{5} \mathrm{H}_{4}\right)$, 6.99-8.32 (m, $\left.14 \mathrm{H}, \mathrm{ArH}^{\prime} \mathrm{s}\right)$ ppm. Anal. Calcd. for $\mathrm{C}_{46} \mathrm{H}_{38} \mathrm{Fe}_{2} \mathrm{~N}_{8} \mathrm{~S}_{2}$ : C, 62.88\%; H, 4.36\%; N, $12.75 \%$. Found: C, $62.84 \% ; \mathrm{H}, 4.39 \%$; N $12.77 \%$.

1,4-Bis-(5-(1-(phenylethylidenehydrazono)-4-phenyl-4,5-dihydro-1,3,4-thiadiazol-2-yl)benzene (12). Brown crystalline solid from EtOH/DMF; Yield (71\%); >300 ${ }^{\circ} \mathrm{C}$; IR ( $\left.\mathrm{cm}^{-1}\right)$ (KBr): 3100, 3053 (C-H Ar), 1615 $(\mathrm{C}=\mathrm{N}) ;{ }^{1} \mathrm{H}-\mathrm{NMR}\left(300 \mathrm{MHz}, \mathrm{DMSO}-d_{6}\right): \delta 2.52\left(\mathrm{~s}, 6 \mathrm{H}, 2 \mathrm{CH}_{3}\right), 7.49-8.51\left(\mathrm{~m}, 14 \mathrm{H}, \mathrm{ArH}^{\prime} \mathrm{s}\right) \mathrm{ppm}$. Anal. Calcd. for $\mathrm{C}_{32} \mathrm{H}_{30} \mathrm{~N}_{8} \mathrm{~S}_{2}$ : C, $68.86 \% ; \mathrm{H}, 4.56 \%$;, $16.91 \%$. Found: $\mathrm{C}, 68.85 \% ; \mathrm{H}, 4.58 \% ; \mathrm{N}, 16.89 \%$.

\subsection{Synthesis of $[1,3,4]$ Thiadiazolo[2,3-c:5,4-c']bis([1,2,4]triazines) (17a-c)}

To 2,5-dihydrazinyl-1,3,4-thiadiazole 13 ( $5 \mathrm{mmol}, 0.73 \mathrm{~g}$ ) and the appropriate hydrazonoyl halides $14(10 \mathrm{mmol})$ in dioxane $(50 \mathrm{~mL})$ was added triethylamine $(1.4 \mathrm{~mL}, 10 \mathrm{mmol})$ at room temperature. The reaction mixture was heated under reflux until all the starting material was consumed ( 9 $\mathrm{h}$, monitored by TLC). The solvent was evaporated and the residue was triturated with $\mathrm{MeOH}$. The formed solid was filtered and recrystallized from DMF to give compounds $\mathbf{1 7 .}$

4,7-Dimethyl-3-((E)-p-tolyldiazenyl)-8-(p-tolyldiazenyl)-2,9-dihydro-[1,3,4]thiadiazolo[2,3-c:5,4-c']bis([1,2,4]triazine) (17a). Yellow crystalline solid from EtOH/DMF; Yield (85\%); m.p. $275^{\circ} \mathrm{C}$; IR $\left(\mathrm{cm}^{-1}\right)(\mathrm{KBr}): 3259(\mathrm{~s}, 2 \mathrm{H}$, $\mathrm{NH}), 1610(\mathrm{C}=\mathrm{N}) ;{ }^{1} \mathrm{H}-\mathrm{NMR}\left(300 \mathrm{MHz}, \mathrm{DMSO}-d_{6}\right): \delta 2.58\left(\mathrm{~s}, 6 \mathrm{H}, 2 \mathrm{CH}_{3}\right), 3.53\left(\mathrm{~s}, 6 \mathrm{H}, 2 \mathrm{CH}_{3}\right), 6.99-7.91$ 
(m, 8H, ArH's), $11.14(\mathrm{~s}, 2 \mathrm{H}, 2 \mathrm{NH})$ ppm. Anal. Calcd. for $\mathrm{C}_{22} \mathrm{H}_{22} \mathrm{~N}_{10} \mathrm{~S}: \mathrm{C}, 57.63 \% ; \mathrm{H}, 4.84 \%$; N, 30.55\%. Found: $\mathrm{C}, 57.66 \% ; \mathrm{H}, 4.89 \% ; \mathrm{N}, 30.51 \%$.

4,7-Dimethyl-3,8-bis(phenyldiazenyl)-2,9-dihydro-1,3,4-thiadiazolo[2,3-c:5,4-c]bis([1,2,4]triazine) (17b). Red crystalline solid from EtOH/DMF; Yield (73\%); m.p. $177^{\circ} \mathrm{C}$; IR $\left(\mathrm{cm}^{-1}\right)(\mathrm{KBr}): 3259$ (s, 2H, NH), 1610 $(\mathrm{C}=\mathrm{N}) ;{ }^{1} \mathrm{H}-\mathrm{NMR}\left(300 \mathrm{MHz}, \mathrm{DMSO}-d_{6}\right): \delta 2.58\left(\mathrm{~s}, 6 \mathrm{H}, 2 \mathrm{CH}_{3}\right), 7.11-8.01(\mathrm{~m}, 11 \mathrm{H}, \mathrm{ArH}$ 's), $11.17(\mathrm{~s}, 2 \mathrm{H}$, 2NH) ppm; ${ }^{13} \mathrm{C}-\mathrm{NMR}\left(300 \mathrm{MHz}, \mathrm{DMSO}-d_{6}\right): \delta 25.54,114.70,115.45,122.15,123.32,124.04,129.47$, 129.47, 130.01, 142.34 and 194.68 ppm. Anal. Calcd. for $\mathrm{C}_{20} \mathrm{H}_{18} \mathrm{~N}_{10} \mathrm{~S}: \mathrm{C}, 55.80 \% ; \mathrm{H}, 4.21 \% ; \mathrm{N}, 32.54 \%$. Found: $\mathrm{C}, 55.83 \% ; \mathrm{H}, 4.24 \% ; \mathrm{N}, 32.58 \%$.

3-((4-Chlorophenyl)diazenyl)-8-((E)-(4-chlorophenyl)diazenyl)-4,7-dimethyl-2,9-dihydro-[1,3,4]thiadiazolo[2,3c:5,4-c']bis([1,2,4]triazine) (17c). Yellow crystalline solid from EtOH/DMF; Yield (68\%); m.p. $245^{\circ} \mathrm{C}$; IR $\left(\mathrm{cm}^{-1}\right)(\mathrm{KBr}): 3259(\mathrm{~s}, 2 \mathrm{H}, \mathrm{NH}), 1610(\mathrm{C}=\mathrm{N}) ;{ }^{1} \mathrm{H}-\mathrm{NMR}\left(300 \mathrm{MHz}, \mathrm{DMSO}-d_{6}\right): \delta 2.58\left(\mathrm{~s}, 6 \mathrm{H}, 2 \mathrm{CH}_{3}\right)$, 7.23-8.24 (m, 8H, ArH's), 11.19 (s, 2H, 2NH) ppm. Anal. Calcd. for $\mathrm{C}_{20} \mathrm{H}_{16} \mathrm{Cl}_{2} \mathrm{~N}_{10} \mathrm{~S}: \mathrm{C}, 48.10 \% ; \mathrm{H}$, $3.23 \%$; N, 28.05\%. Found: C, 48.15\%; H, 3.21\%; N, 28.057\%.

\subsection{Synthesis of Pyrazolothiadiazole (22) and (23)}

A mixture of 2,5-dihydrazinyl-1,3,4-thiadiazole $\mathbf{1 3}(5 \mathrm{mmol}, 0.73 \mathrm{~g}$ ) and acetylacetone $\mathbf{1 8}$ (10 mmol, $1.00 \mathrm{~g})$ or ethyl acetoacetate $19(10 \mathrm{mmol}, 1.30 \mathrm{~g})$ in glacial acetic acid $(20 \mathrm{~mL})$ was refluxed for $6 \mathrm{~h}$. After cooling, the precipitate was collected by filtration and crystallized from the appropriate solvent to afford compounds 22 and 23.

2,5-Bis(3,5-dimethyl-1H-pyrazol-1-yl)-1,3,4-thiadiazole (22). Pale yellow crystalline solid from EtOH/DMF; Yield (65\%); m.p. $183{ }^{\circ} \mathrm{C}$; IR ( $\left.\mathrm{cm}^{-1}\right)(\mathrm{KBr}): 3053$ (C-H Ar), 1610 (C=N); ${ }^{1} \mathrm{H}-\mathrm{NMR}\left(300 \mathrm{MHz}, \mathrm{DMSO}-d_{6}\right)$ : $\delta 3.50\left(\mathrm{~s}, 12 \mathrm{H}, 2 \mathrm{CH}_{3}\right), 6.18$ (s, 2H, 2 azomethine) ppm, ${ }^{13} \mathrm{C}-\mathrm{NMR}\left(300 \mathrm{MHz}, \mathrm{DMSO}-d_{6}\right): \delta 17.31,114.61$, 151.95, 163.05, 188.10 ppm. Anal. Calcd. for $\mathrm{C}_{12} \mathrm{H}_{14} \mathrm{~N}_{6} \mathrm{~S}: \mathrm{C}, 52.54 \% ; \mathrm{H}, 5.14 \%$;, $30.63 \%$. Found: $\mathrm{C}$, $52.56 \% ; \mathrm{H}, 5.17 \% ; \mathrm{N}, 30.61 \%$.

1,1'-(1,3,4-Thiadiazole-2,5-diyl)bis(3-methy-1H-pyrazol-5(4H)-one) (23). Pale yellow crystalline solid from EtOH/DMF; Yield (71\%); m.p. $275{ }^{\circ} \mathrm{C}$; IR ( $\left.\mathrm{cm}^{-1}\right)(\mathrm{KBr}): 3055(\mathrm{C}-\mathrm{H} \mathrm{Ar}), 1715(\mathrm{C}=\mathrm{O}), 1615(\mathrm{C}=\mathrm{N})$; ${ }^{1} \mathrm{H}-\mathrm{NMR}\left(300 \mathrm{MHz}, \mathrm{DMSO}-d_{6}\right): \delta 3.17\left(\mathrm{~s}, 6 \mathrm{H}, 2 \mathrm{CH}_{3}\right), 3.84\left(\mathrm{~s}, 4 \mathrm{H}, 2 \mathrm{CH}_{2}\right) \mathrm{ppm}$. Anal. Calcd for $\mathrm{C}_{10} \mathrm{H}_{10} \mathrm{~N}_{6} \mathrm{O}_{2} \mathrm{~S}: \mathrm{C}, 43.16 \% ; \mathrm{H}, 3.62 \%$; N, 30.20\%. Found: $\mathrm{C}, 43.19 \% ; \mathrm{H}, 3.64 \% ; \mathrm{N}, 30.24 \%$.

\section{Conclusions}

In conclusion, the studied reactions provide a facile new route for synthesized thiadiazoles, bisthiadiazoles, pyrazolothiadiazoles, and thiadiazolotriazines via the utility of hydrazonoyl halides and 2,5-dihydrazinyl-1,3,4-thiadiazole. The final products were identified by different techniques, such as elemental analysis and FT-IR, NMR, mass spectrometry, and alternate synthesis whenever possible.

Acknowledgments: The financial support by the Deanship of Scientific Research (Project Number 160035), King Faisal University, Saudi Arabia, is gratefully acknowledged.

Author Contributions: Abdelwahed R. Sayed planned the synthesis thiadiazoles, bisthiadiazoles, thiadiazolotriazines and pyrazolothiadiazoles. Abdelwahed R. Sayed and Shar Saad Al-Shihry performed the analyses and drafted the manuscript.

Conflicts of Interest: The authors declare no conflict of interest.

\section{References}

1. Shawali, A.S.; Sayed, A.R.; Zayed, M.M. A convenient method for synthesis of bis-2,2'-(1,3,4-thiadiazole) and bis-3,3'-(1,2,4-triazole) derivatives. J. Sulfur Chem. 2011, 32, 311-314. [CrossRef]

2. Sayed, A.R. Synthesis of 1,3,4-thiadiazines, bis-1,3,4-thiadiazoles, $[1,2,4]$ triazino[3,4- $b][1,3,4]$ thiadiazine, thiazolines from carbonothioic dihydrazide. Tetrahedron 2012, 68, 2784-2789. [CrossRef]

3. Sayed, A.R. Synthesis of novel bis-thiadiazoles, bis-triazoles and polypyrazole derivatives based on hydrazonoyl halides. Tetrahedron 2013, 69, 5293-5298. [CrossRef] 
4. Shawali, A.S. 1,3,4-Thiadiazoles of pharmacological interest: Recent trends in their synthesis via tandem 1,3-dipolar cycloaddition: Review. J. Adv. Res. 2014, 5, 1-17. [CrossRef] [PubMed]

5. Sayed, A.; Zaki, Y.; Aish, E. A convenient route for the synthesis of new thiadiazoles. Turk. J. Chem. 2016, 40, 184-191. [CrossRef]

6. Mann, J.; Baron, A.; Opoku-Boahen, Y.E.; Johansson, G.; Parkinson, L.R.; Kelland, S.; Neidle, A. A New Class of Symmetric Bisbenzimidazole-Based DNA Minor Groove-Binding Agents Showing Antitumor Activity. J. Med. Chem. 2001, 44, 138-144. [CrossRef] [PubMed]

7. Ujjinamatada, R.K.; Baier, A.; Borowski, P.; Hosmane, R.S. An analogue of AICAR with dual inhibitory activity against WNV and HCV NTP ase/helicase: Synthesis and in vitro screening of 4-carbamoyl-5-(4,6-diamino-2,5-dihydro-1,3,5-triazin-2-yl)imidazole-1- $\beta$-D-ribofuranoside. Bioorg. Med. Chem. Lett. 2007, 17, 2285-2288. [CrossRef] [PubMed]

8. Rostom, S.A.F.; Ashour, H.M.A.; El Razik, H.A.; El Fattah, A.F.; El-Din, N.N. Azole antimicrobial pharmacophore-based tetrazoles: Synthesis and biological evaluation as potential antimicrobial and anticonvulsant agent. Bioorg. Med. Chem. 2009, 17, 2410-2422. [CrossRef] [PubMed]

9. Adams, J.L.; Boehm, J.C.; Gallagher, T.F.S.; Kassis, E.F.; Webb, R.; Hall, M.; Sorenson, R.; Garigipati, G.D.E.; Lee, J.C. Pyrimidinylimidazole Inhibitors of p38: Cyclic N-1 Imidazole Substituents Enhance p38 Kinase Inhibition and Oral Activity. Bioorg. Med. Chem. Lett. 2001, 11, 2867-2870. [CrossRef]

10. Steel, P.J. Aromatic biheterocycles: Syntheses, structures, and properties. Adv. Heterocycl. Chem. 1997, 67, $1-117$.

11. Weinstock, L.M.; Shankai, I. Comprehensive Heterocyclic Chemistry; Potts, K.T., Ed.; Pergmann: Oxford, UK, 1984; Volume 6.

12. Glossman, M.D. Local and nonlocal density functional calculations of the molecular structure of isomeric thiadiazoles. J. Mol. Struct.: THEOCHEM 1997, 390, 67-78. [CrossRef]

13. Mitnik, D.G.; Lucero, A.M. HF and DFT calculations of the molecular structure of isomeric thiadiazole dioxides. J. Mol. Struct.: THEOCHEM 2001, 549, 285-288. [CrossRef]

14. Krygowski, T.M. Crystallographic studies of inter- and intramolecular interactions reflected in aromatic character of $\pi$-electron systems. J. Chem. Inf. Comput. Sci. 1993, 33, 70-73. [CrossRef]

15. Arakelian, A.N.; Davis, K.E. 2,5-Dimercapto-1,3,4-thiadiazole derivatives and lubricants containing them. U.S. Patent 42 46126, 1981, 20 January 1981.

16. Abdelhamid, A.O.; Sallam, M.M.M.; Amer, S.A. Reaction with hydrazonoyl halides. Part 32 [1]: Reaction of C-acyl-N-(3-phenyl-5-pyrazolyl)hydrazonoyl chlorides with potassium thiocyanate and synthesis of some new 2,3-dihydro-1,3,4-thiadiazoles and slenadiazoles. Heteroat. Chem. 2001, 12, 468-474. [CrossRef]

17. Antolini, L.; Benedetti, A.; Fabretti, A.C.; Giusti, A.; Menziani, M.C. Crystal and molecular structure of bis(2-amino-5-methyl-1,3,4-thiadiazole- $N^{3}$ )dibromomercury(II). A spectroscopic study and INDO calculations. J. Chem. Soc. Dalton Trans. 1988, 1075-1077. [CrossRef]

18. Dogan, H.N.; Duran, A.; Rollas, S.; Sener, G.; Uysal, M.K.; Gulen, D. Synthesis of new 2,5-Disubstituted-1,3,4-thiadiazoles and preliminary evaluation of anticonvulsant and antimicrobial activities. Bioorg. Med. Chem. 2002, 10, 2893-2898. [CrossRef]

19. Palaska, E.; Sahin, G.; Kelicen, P.; Turlu, N.T.; Altinok, G. Synthesis and anti-inflammatory activity of 1-acylthiosemicarbazides, 1,3,4-oxadiazoles, 1,3,4-thiadiazoles and 1,2,4-triazole-3-thione. Il Farmaco 2002, 57, 101-107. [CrossRef]

20. Karakus, S.; Rollas, S. Synthesis and antituberculosis activity of new N-phenyl- $N^{\prime}$-[4-(5-alkyl/arylamino-1,3,4thiadiazole-2-yl)phenyl]thioureas. Il Farmaco 2002, 57, 577-581. [CrossRef]

21. Terzioglu, N.; Gursoy, A. Synthesis and anticancer evaluation of some new hydrazone derivatives of 2,6-dimethylimidazo[2,1-b][1,3,4]thiadiazole-5-carbohydrazide. Eur. J. Med. Chem. 2003, 38, 781-786. [CrossRef]

22. Bassam, A.; Khalili, F.I.; Hamadneh, I.; Al-Dujaili, A.H. Synthesis and characterization of new polyamides containing symmetrical and unsymmetrical thiadiazole rings. Fibers Polym. 2016, 17, 166-173.

23. Dawood, K.M.; Gomha, S.M. Synthesis and Anti-cancer Activity of 1,3,4-Thiadiazole and 1,3-Thiazole Derivatives Having 1,3,4-Oxadiazole Moiety. J. Heterocycl. Chem. 2015, 52, 1400-1405. [CrossRef]

24. Mukund, P.S.; Levi, M.S.; Takahiro, S. Enantioselective 1,3-Dipolar Cycloaddition of Nitrile Imines to $\alpha$-Substituted and $\alpha, \beta$-Disubstituted $\alpha, \beta$-Unsaturated Carbonyl Substrates: A Method for Synthesizing Dihydropyrazoles Bearing a Chiral Quaternary Center. Adv. Synth. Catal. 2006, 348, 2371-2375. 
25. Audrieth, L.F.; Scott, E.S.; Kippur, P.S. Hydrazine derivatives of the carbonoic and thiocarbonic acids. I. The preparation and properties of thiocarbohydrazide. J. Org. Chem. 1954, 19, 733-741. [CrossRef]

26. Ismail, K.Z. Synthesis and physicochemical studies of metal complexes of ferrocene Schiff base derivatives. Transit. Met. Chem. 1997, 22, 565-569. [CrossRef]

27. Stille, I.J.K.; Harris, F.W.; Bedford, M.A. Phenyl substituted dipyrazoles: 1,3-dipole addition reactions of sydnones and nitrilimines. J. Heterocycl. Chem. 1966, 3, 155-157. [CrossRef]

28. Lin, A.J.; Klayman, D.L.J. A facile synthesis of unsymmetrical heterocyclic azines by cyclodesulfurization: Reaction of methyl arylalkylidenehydrazinecarbodithioates with diamines. J. Heterocycl. Chem. 1985, 22, 1-4. [CrossRef]

29. Jackman, D.E.; Jackman, D.E. Structure and synthesis of the metribuzin blue dye. Synthesis and reactions of 2,5-dihydrazino-1,3,4-thiadiazole and its derivatives. J. Heterocycl. Chem. 1990, 27, 1053-1057. [CrossRef]

30. Dubenko, R.G.; Gorbenko, E.F. Substituted glyoxalic acids. XI. Synthesis of arylhydrazones of acid chlorides of substituted glyoxalic acid and reactions with amines and hydrazine. Zh. Org. Khim. 1968, 4, 634-638.

Sample Availability: Samples of the compounds 12, 17a-c, 22 and 23 are available from the authors. 